Ann. Zootech., I967, 16 (I), 4I-63.

\title{
COMPARAISON DE LA VALEUR DE DIVERS TYPES DE CROISEMENT INDUSTRIEL POUR LA PRODUCTION D'AGNEAUX DE BOUCHERIE.
}

\author{
II. - VALEUR BOUChÈre DES AGNEAUX \\ J.-C. FLAMANT, P. CATTIN-VIDAL, J. POLY \\ aver la collaboration technique de Nathalie Ryscinexiov \\ Station centrale de Génétique animale, \\ Centre national de Recherches zoolechniques, 78-Jouy-en-Josas
}

SOMMAIRE

Les expériences menées à l'Abbaye d'Aiguebelle (1)rôme) et ì l'École d'Agriculture de Marmilhat (Puy-de-Dôme) ont permis la comparaison de différents types d'agneaux (croisés ou de race pure), pour la production de carcasses de $15 \mathrm{~kg}$. L'analyse a confirmé l'influence du sexe sur la qualité des carcasses : les carcasses femelles ont un meilleur rendement, une meilleure conformation, une ossature plus légère et un tissu gras plus développé que les carcasses mâles. L'influence du mode d'élevage se manifeste sur le poids de la carcasse et sur l'importance du tissu osseux, les agneaux élevés simples ayant un rendement en carcasse meilleur, et un os canon plus lourd que les agneaux doubles.

Ia part d'influence de chacun des facteurs sur les variations du poids des carcasses, leur conformation et leur composition tissulaire a été déterminée. Les facteurs génétiques affectent essentiellement la conformation des carcasses et le développement du squelette. Les variations de ces 2 caractères constituent les seuls éléments de différenciation des agneaux de race pure par rapport aux agneaux croisés d'une part, et entre types d'agneaux croisés d'autre part. L'utilisation de béliers de races dites améliorées, en croisement avec des brebis de races montagnardes rustiques se manifeste che $z$ leurs descendants principalement par une amélioration de la conformation. L'utilisation de béliers Southdown (ou Charmoise) conduit en outre aux carcasses les mieux conformées, d'ossature plus légère mais avec un tissu adipeux plus important.

Lc croisement Berrichon, qui s'est révélé préférable au croisement Southdown pour la production précoce d'agneaux de plus de $30 \mathrm{~kg}$ conduit à des carcasses dont la conformation et la composition tissulaire sont intermédiaires entre celles des carcasses d'agneaux de race pure et celles des carcasses croisées Southdown. Compte tenu des conditions locales de commercialisation et pour la production d'agneaux de $30 \mathrm{~kg}$, l'utilisation des béliers de races de grand format du type Berrichon $d u$ Cher ou Ile-de-France est à recommander en croisement industriel avec les brebis de races Préalpes et Bize. 


\section{A. - INTRODUCTION}

I.es expériences de croisement industriel menées durant 5 ans de 1957 à Ig6I à l'Abbaye d'Aiguebelle (Irôme) et à l'Ácole régionale d'Agriculture de Marmilhat (Puy-de-Iôme) ont déjà été interprétées dans des publications précédentes par Desvignes et I)Arpoux (Ig64) pour la valeur d'élevage des femelles, et par 1)ESvignes, Cat'TiN-VIDal, et POIY (I965) pour la croissance pondérale des agneaux de la naissance à l'abattage.

Les brebis élevées à Aiguebelle appartenaient à la race Préalpes, et à Marmilhat à la race Bizet. Ces brebis ont été mises à la lutte avec des béliers des races Préalpes, Southdown, Berrichonne du Cher, Ile-de-France et Charmoise à Aiguebelle; Bizet, Southdown, Berrichonne du Cher et Ile-de-France à Marmilhat.

Les agneaux de race pure ou produits des croisements ont été abattus à un poids voisin de $27 \mathrm{~kg}$ à Marmilhat et de $30 \mathrm{~kg}$ à Aiguebelle (1).

1)ESVIgNes, Ca'Tin-VIDAI, et POIY $\left(\mathrm{Ig}^{6} 65\right)$ ont montré l'importance du choix de la race des béliers pour la croissance en fin d'engraissement des agneaux croisés. Ainsi l'utilisation de béliers des races Berrichonne $d u$ Cher et Ile-de-France conduit à des agneaux dont la croissance est linéaire de la naissance à l'abattage et parviennent plus rapidement au poids désiré, alors que les croisés Southdor $n$ accusent nettement une diminution du gain de poids journalier après l'âge de 2 mois.

Lans cette deuxième partie nous nous proposons d'étudier maintenant ces différents types de croisement du point de vue de la qualité des carcasses appréciée par des mensurations.

\section{B. - .... VALEUR BOUCHÈ̀RE DES CARCASSES I. - MAT́T́RIEI, ET TECHNIQUES}

L'étude porte sur les données complètes de $45 \mathrm{I}$ carcasses dont la répartition en 6 agnelages est décrite dans le tableau $\mathrm{I}$. Le tablean 2 rapporte par ailleurs la succession chronologique et l'identification des agnelages contrôlés dans charque exploitation.

Les mesures suivantes ont été effectuées sur chacune des carcasses (fig. I) :

a) Pesée des carcasses.

Après abattage des agneaux, les carcasses sont maintenues 24 heures en chambre froide. La pesée est effectuée au bout de ce délai avec une balance assurant une précision de roog (tête, 4 pieds et rognons prélevés). La carcasse est alors découpée transversalement entre la ${ }_{1}$ re et la $2^{\mathrm{e}}$ vertèbre lombaire : la partic antérieure constitue le "colfre " et la partie postérieure le "baron " dont les pesées sont elfectuées avce la même précision cque celle de la carcasse. I'n calcue est pris au niveau de la section du muscle longissimus dorsi, sur lequel sont effectuées ensuite les mesures droite et gauche d'épaisseur du gras dorsal, de largeur, longueur et surface de la section du longissimus dorsi.

b) Hesurcs d'importance du tissu adipeux.

Deux mesures ont été retenues : le porids du gras de rognon (Boccarb et Donont, ig6o) et l'épaisseur du gras du dessus de la côte ( $C^{\prime}$ : moyenne des mesures droite et gauche).

(1) 28 à $32 \mathrm{~kg}$ à Aiguebelle (ct pour le premier agnelage de Marmilhat.) 25 à $29 \mathrm{~kg}$ à Marmilhat ( $2^{\mathrm{e}}$ et $3^{\circ}$ agnelarges). 
TABLEAU I

Tableau des effectifs par facteurs de classement intra-agnelage

\begin{tabular}{|c|c|c|c|c|c|c|c|c|}
\hline \multirow{2}{*}{ 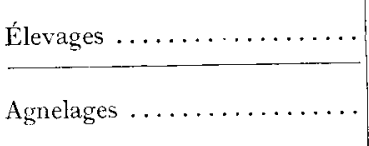 } & \multicolumn{4}{|c|}{ Aiguebelle } & \multicolumn{4}{|c|}{ Marmilhat } \\
\hline & I & II & IV & $\begin{array}{c}3 \text { agnelages } \\
\text { combinés }\end{array}$ & 1 & II & III & $\begin{array}{c}3 \text { agnelages } \\
\text { combinés }\end{array}$ \\
\hline Total $\ldots \ldots \ldots \ldots \ldots \ldots \ldots$ & 71 & 80 & 129 & 196 & 43 & 74 & 44 & 132 \\
\hline Race du Père & & & & & & & & \\
\hline Soulhdown $\ldots \ldots \ldots \ldots \ldots \ldots$ & 23 & 30 & $1^{\prime} t_{\mathrm{k}}$ & 67 & 31 & 30 & 12 & 73 \\
\hline Bizet.$\ldots \ldots \ldots \ldots \ldots \ldots \ldots$ & - & 一 & -- & 一 & - & 16 & 13 & $一$ \\
\hline Préalpes................. & 8 & 17 & 一 & - & $一$ & - & $一$ & - \\
\hline Berrichonne du Cher......... & 25 & 15 & 89 & 129 & 12 & 28 & 19 & 59 \\
\hline Charmoise................... & 15 & 28 & - & 一 & 一 & - & 一 & - \\
\hline Ile-de-France............... & - & - & 26 & - & - & -- & 一 & - \\
\hline Mode d'élevage & & & & & & & & \\
\hline Simple $\ldots \ldots \ldots \ldots \ldots \ldots$ & 30 & 61 & $9 !$ & 136 & 27 & 39 & 27 & 75 \\
\hline Double...$\ldots \ldots \ldots \ldots \ldots$ & 41 & 229 & 35 & 60 & 16 & 35 & 17 & 57 \\
\hline Sexe & & & & & & & & \\
\hline Mâle $\ldots \ldots \ldots \ldots \ldots \ldots \ldots \ldots$ & 36 & 51 & 61 & 94 & 29 & 46 & 32 & 79 \\
\hline Femelle...$\ldots \ldots \ldots \ldots \ldots$ & 35 & 39 & 68 & 102 & $1 / 4$ & 28 & 12 & 53 \\
\hline
\end{tabular}

TABLEAU 2

Chronologie des agnelages étudiés

\begin{tabular}{|c|c|c|c|}
\hline Élevage & $N^{\circ}$ d'agnelage & ípoque d'agnelage & Année \\
\hline Aiguebelle & $\begin{array}{c}\text { I } \\
\text { II } \\
\text { IV }\end{array}$ & $\begin{array}{c}\text { avril-mai } \\
\text { novembre-décembre } \\
\text { août-septembre }\end{array}$ & $\begin{array}{l}1958 \\
1958 \\
1959\end{array}$ \\
\hline Marmilhat & $\begin{array}{r}\text { I } \\
\text { II } \\
\text { III }\end{array}$ & $\begin{array}{c}\text { octobre-décembre } \\
\text { octobre-décembre } \\
\text { septembre-décembre }\end{array}$ & $\begin{array}{l}1957 \\
1958 \\
1959\end{array}$ \\
\hline
\end{tabular}


c) Mesures d'importance du tissu osseux. sures :

L'importance du tissu osseux dans la carcasse est évaluée à l'aide de deux catégories de me-
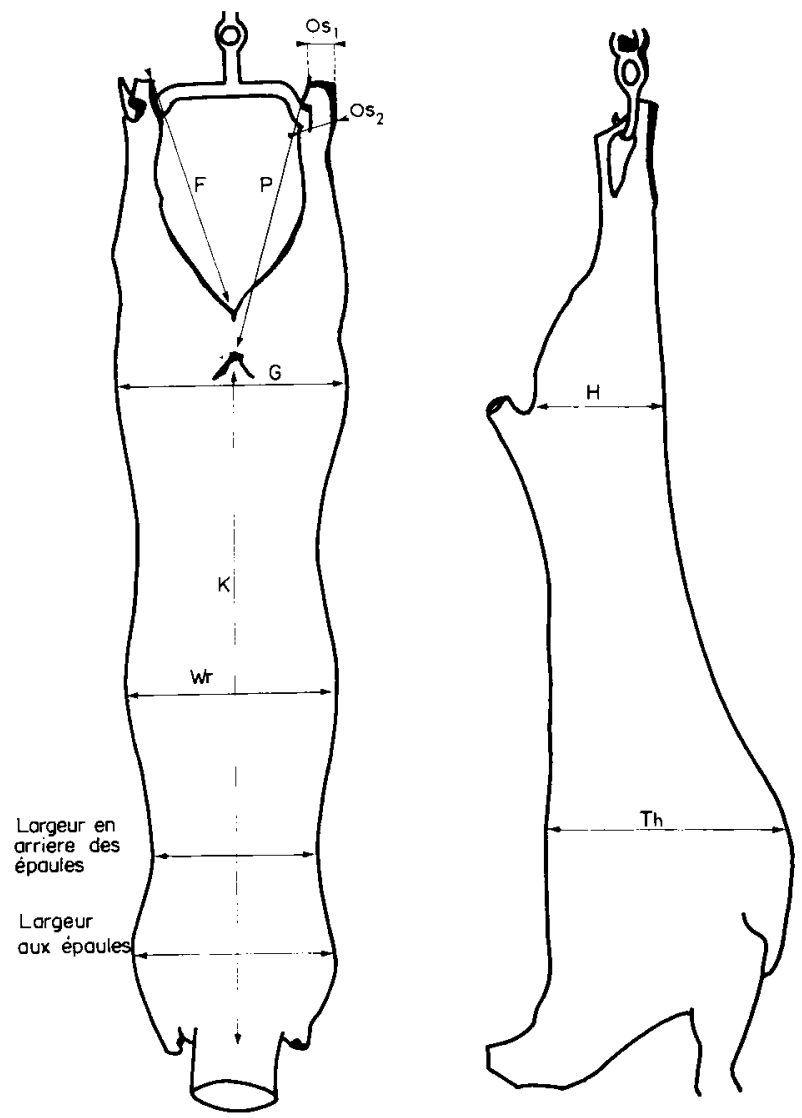

Fig. 1. - Mensuralions effectuies sur les carcasses non découpées

$1^{\circ}$ Évaluation par le poids de l'os métacarpe (os canon) avec une balance précise à a g près et par le poids des 4 pieds avec une précision de $\pm 5 \mathrm{~g}$ (PaLSSON, 1939). Cette dernière évaluation est moins valable puisque outre les 4 os canons, les 4 pieds comprennent le poids de la peau, des phanères, des tendons, du gras et du tissu musculaire.

$2^{\mathrm{o}}$ Évaluation par le diumetre des us :

$$
\begin{aligned}
& \text { épaisseur de la crosse }\left(\mathrm{O}_{81}\right) \\
& \text { épaisseur du condyle }\left(O_{82}\right) \text {. }
\end{aligned}
$$

Ces 2 mesures sont effectuées sur chaque $1 / 2$ carcasse. Leur somme constitue l'indice "Os " proposé par BOCCARI, DUMONT et l'EYRON (I958).

d) Caractéristiques de développement des masses musculaires.

Le développement des muscles est évalué par des mesures prises sur les 2 morceaux nobles de la carcasse : le gigot et le filet (STARKle et JotBERT, 1961).

- largeur de la carcasse aux gigots ( $(\mathrm{i})$ et épaisseur des gigots (H) les carcasses étant suspendues à écartement de tinets constant $(22 \mathrm{~cm})$;

- longueur $\left(\mathrm{A}^{\prime}\right)$, largeur $\left(\mathrm{B}^{\prime}\right)$ et surface de la section du muscle longissimus dorsi entre la ${ }^{\text {re }}$ et la $2^{2}$ vertèbre lombaire (moyenne des mesures droite et gauche). 
e) Longueur des rayons osseux et conformation des gigots.

longueur de l'os canon (M),

longueur crosse-périnée (F),

longueur crosse-symphyse $(\mathrm{P})$.

Cette dernière mesure a été rctenue par BaRToN, Puilitips et Ciarke (I949) de préférence à $F$ puur exprimer la conformation des gigots.

f) Dimension générale des carcasses :

largeur aux épaules

largeur en arrière des épaules

largeur de poitrine $(\mathrm{Wr})$

hauteur de poitrine (Th)

longueur quene-cou $(\mathrm{K})$.

FLAMANT et BOCcar1) (I966) ont effectué une revue bibliographique critique sur la représentativité des mesures sur carcasses comme indices de qualité et considèrent qu'en première approximation, le poids de l'os canon et le poids du gras de rognon permettent d'estimer la composition corporelle des carcasses, tandis que la longueur $F^{*}$ caractérisé parfaitement la conformation.

\section{Wéthodologie statistique}

Afin de déterminer l'effet de la race du père sur les caractéristiques des carcasses indépend:mment d'autres facteurs, nous avons dû prendre en considération lè sexe, le mode d'élevage et le poids à l'abattage des agneaux.

T.e modèle mathémat ique utilisé tient compte des différents " effets principaux n et du poids à l'abattage. Il s'applique ì des schémas à plusieurs voies, avec des nombres inégaux d'observations dans charque classe, et sans interaction (KeMrTHORNe, 1958).

$$
\mathrm{Y}_{i j k l}=\mu+\mathbf{R}_{i}+\mathrm{M}_{j}+\mathrm{S}_{k}+b \mathrm{X}_{i j k l} \div e_{i j k l}
$$

$Y_{i j k l}$ est une observation du $l$ ième agneau de sexe $k$, de mode d'élevage $j$ et issu d'un bélier de race $i$.

:L est une constante commune is tous les agneaux.

$\mathrm{R}_{i} \quad$ est un coefficient particulier à la $i$ eme race de bélicr.

$\mathrm{MI}_{j} \quad$ est un coefficient particulier au $i$ ème mode d’élevage.

$s_{k} \quad$ est un coefficient particulier au $k$ ième sexe.

$X_{i j k l}$ est le poids a l'abattage de l'agneau considéré (covariable).

$b \quad \epsilon s t$ un coefficient de régression partielle qui lie $\mathrm{I}$ à la covariable.

eijht est une variable aléatoire de moyenne nulle et de variance $\sigma_{e}{ }^{2}$, variance résiduelle sou. mise aux influences autres rque celles des facteurs contrôlés.

I.es hypothèses suivantes ont été faites pour rendre valable le schéma décrit :

Hypothèse I : nullité des interactions entre les facteurs de classement.

Hypothèse $1 \mathrm{I}$ : indépendance entre la covariable et les facteurs de classement.

Lat résolution des systènes linéaires ainsi constitués permet d'obtenir, pour chaque variable intra-agnelage, les estimées de $\mu, \mathrm{R}_{i}, \mathrm{M}_{j}, \mathrm{~S}_{k}$ et $b$, et de calculer ensuite les réductions du type C ( $\mu, R, M, S)$, carrés moyens dus aux facteurs analysés (KEMPTIORNE, 1958). La signification des effets cles facteurs de classement et du poids à l'abat tage sur les variations étudiées a été déterminée par analyse de variance (I)FSvignes, CATTIN-VIDAL, POLY; i 965 ). Les coefficients $\mathrm{R}_{i}$, obtenus pour les races en présence, ont été comparés deux à deux et leurs différences observées ont été testées par la méthode de Duncan, étendue aux schémas non orthogonaux (Kramer, 1956) en assimilant $\mathrm{R}_{i}$ a une moyenne.

Par ailleurs la comparaison des performances du croisement " bélier Southdown $\times$ brebis de race rustique "avec les résultats obtenus par l'emploi de béliers de la race Berrichonne du (her nous a amené i regrouper intra-élevages les domnées relatives à ces 2 types de croisement. L'analyse a donc été conduite selon le schéma mathématique suivant tenant compte d'un $4^{\mathrm{e}}$ effet principal, l'effet agnelage, assimilable à l'effet année :

$$
\mathrm{Y}_{i j k l m}=\mu+\mathrm{R}_{i}+\mathrm{H}_{j}+S_{k}+\mathrm{A}_{l}+b \mathrm{X}_{i j k l m} \div e_{i j k l m}
$$

où Yijklm est une observation du mième agneau, né la lième année, de sexe $k$, de mode d'élevage $j$ 
et ayant pour père un bélier de race $i$. A est un coefficient particulier à la $l$ ième année. (Ce facteur amnée est en fait un facteur agnelage à Aiguebelle, oì les 2 premiers agnelages ont eu lieu en une seule année.)

Comme pour le premier schéma, nous avons fait les hypothèses I et II d'indépendance des facteurs contrôlés. Les variables nécessitant la découpe n'ont pas été comprises dans ces derniers calculs par suite de l'absence partielle de données. Pour la même raison le poids du gras de rognon n'a pas été compris dans l'analyse de l'élevage de Marmilhat.

ANNE

Mensurations

(moyenne, écart-type, test $F$ de signifi

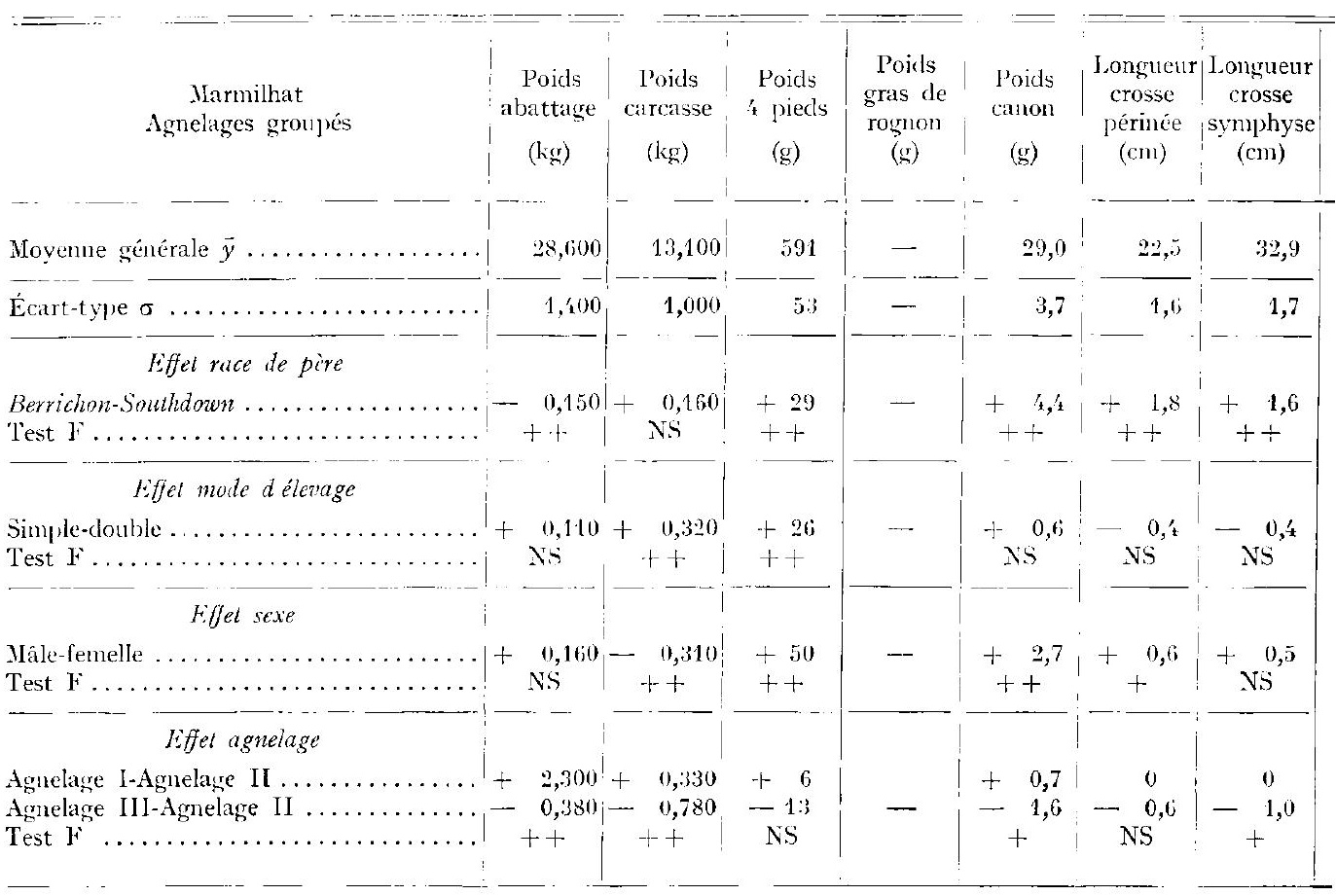

Ns : Effet non signiticatif $(\mathrm{P}>0,05)+$ - Effet significatif $(0,05>\mathrm{P}>0,01)$.

++ : Jiffet hautement significatif $\left(\mathrm{P}^{\prime}<0,01\right)$ Table de Snedecor.

\section{Vérification des hypothèses}

a) Hypothèse I (indépendance des facteurs de classement).

Pour tester la nullité des interactions entre les facteurs de classement nous avons comparé la différence entre le carré moyen "entre cellules " et la réduction totale $C(\mu \mathrm{R}$ M S $(A))$ au carré moyen résiduel. Dans le cas d'un test $F$ significatif aux seuils de probabilité de i p. roo ou de 5 p. roo nous avons testé l'existence d'interactions du premier ordre (entre facteurs de classement pris deux à deux) à l'aide de la méthode des moyennes pondérées décrite par ANDERson et BANCrofT (i952). Sauf cas particuliers qui seront étudiés dans les paragraphes suivants, l'hypothèse d'indépendance a toujours été vérifiée.

b) Hypothèse II (indépendance entre les facteurs de classement et le poids à l'abattage).

La liaison entre les facteurs de variation contrôlés et le poids à l'abattage a été étudiée en utili. sant le schéma mathématique suivant

$$
\mathrm{Z}_{i j k l m}=\mu+\mathrm{R}_{i}+\mathrm{M}_{j}+\mathrm{S}_{k}+\left(\mathrm{A}_{l}\right)+e_{i j k(l) m}
$$


où $Z_{i j k(l) m}$ est le poids à l'abattage du $m$ ième agneau, les autres symboles ayant la même signification que dans les schémas décrits plus haut.

L'influence de l'agnelage est évidemment importante à Marmilhat puisque le poids recherché pour la commercialisation est passé de $30 \mathrm{~kg}$ la première année à $37 \mathrm{~kg}$ les années suivantes. Mais il s'agit d'un effet artificiel dù à l'intervention de l'expérimentateur. En ce qui concerne les autres facteurs du classement, il apparaît que malgré les précautions prises, un biais significatif, pouvant atteindre quelques centaines de grammes, a été introduit par la race du père et le sexe sur le poids à l'abattage. Ce biais peut être considéré comme étant de l'ordre des imprécisions de pesées.

XE I

sur carcasse

cation des effets principaux, valeur de $b$ )

\begin{tabular}{|c|c|c|c|c|c|c|c|c|c|c|}
\hline $\begin{array}{c}\text { Longueur } \\
\text { queue } \\
\text { cou } \\
\text { (cm) }\end{array}$ & $\begin{array}{c}\text { Iargeur } \\
\text { rigot } \\
(\mathrm{cm})\end{array}$ & $\begin{array}{l}\text { Fraisseur } \\
\text { grigot } \\
(\mathrm{cm})\end{array}$ & $\begin{array}{c}\text { Hauteur } \\
\text { poitrine } \\
\text { (cm) }\end{array}$ & $\begin{array}{l}\text { Iargeur } \\
\text { poitrine } \\
\text { (cm) }\end{array}$ & $\begin{array}{l}\text { Largeur } \\
\text { aux } \\
\text { épaules } \\
\text { (cm) }\end{array}$ & $\begin{array}{c}\text { Largeur en } \\
\text { arriére des } \\
\text { éraules } \\
\text { (cm) }\end{array}$ & $\begin{array}{c}O s_{1} \\
\text { ćpaisseur } \\
\text { crosse } \\
(\mathrm{mm})\end{array}$ & $\begin{array}{c}\mathrm{Os}_{2} \\
\text { épaisseur } \\
\text { condyle } \\
\text { (mm) }\end{array}$ & $\begin{array}{l}\text { Indice } \\
\text { "Os" } \\
(\mathrm{mm})\end{array}$ & $\begin{array}{c}\text { II } \\
\text { longueur } \\
\text { canon } \\
\text { (mm) }\end{array}$ \\
\hline 60,9 & 20,2 & $12, k$ & 23,1 & 20,2 & 一 & - & 25,2 & 33,5 & 58,7 & 110,2 \\
\hline 2,5 & 1,2 & 0,6 & 0,9 & 1,3 & - & $\ldots$ & 1,7 & 1,9 & 9,5 & 5,9 \\
\hline $\begin{array}{c}+0,8 \\
\text { NS }\end{array}$ & $\begin{array}{c}+\quad 13,5 \\
++\end{array}$ & $\begin{array}{l}+0,1 \\
\mathrm{NS}\end{array}$ & $\begin{array}{c}+0,5 \\
++\end{array}$ & $\begin{array}{c}+1,0 \\
++\end{array}$ & - & 一 & $\begin{array}{c}+1,8 \\
++\end{array}$ & $\begin{array}{c}+2,1 \\
++\end{array}$ & $\begin{array}{c}+3,9 \\
++\end{array}$ & $\begin{array}{c}+\begin{array}{r}6,3 \\
++\end{array}\end{array}$ \\
\hline $\begin{array}{c}-10,3 \\
\mathrm{NS}\end{array}$ & $\begin{array}{c}+0,3 \\
+\end{array}$ & $\begin{array}{l}+\quad 0,1 \\
\mathrm{NS}\end{array}$ & $\begin{array}{c}-0,4 \\
+\end{array}$ & ${ }^{-+} \mathrm{NS}^{0,3}$ & - & - & $\begin{array}{c}+0,1 \\
\mathrm{NS}\end{array}$ & $\begin{array}{c}+0,6 \\
++\end{array}$ & $\begin{array}{c}+\quad 0,7 \\
+\end{array}$ & $-{ }^{-1,5}$ \\
\hline $\begin{array}{c}+0,3 \\
\times S\end{array}$ & 0 & $\begin{array}{c}-0,2 \\
+\end{array}$ & $\begin{array}{c}+0,2 \\
\mathrm{NS}\end{array}$ & $\begin{array}{c}-0, f_{t}^{\prime} \\
+\end{array}$ & - & - & $\begin{array}{l}+1,5 \\
++\end{array}$ & $\begin{array}{c}+1,9 \\
+\end{array}$ & $\begin{array}{c}+3,{ }^{\prime} \\
\mathrm{NS}\end{array}$ & $\begin{array}{c}+2,1 \\
+\end{array}$ \\
\hline $\begin{array}{c}+0,2 \\
0 \\
\mathrm{NS}\end{array}$ & $\begin{array}{r}+0 \\
-1,4 \\
++\end{array}$ & $\begin{array}{c}-0,3 \\
+0,1 \\
\text { NS }\end{array}$ & $\begin{array}{c}-0,6 \\
-0,7 \\
+t\end{array}$ & $\begin{array}{c}+0,4 \\
+\quad 0,5 \\
\mathrm{NS}\end{array}$ & - & 一 & $\begin{array}{c}+\quad 0,5 \\
-1,6 \\
++\end{array}$ & $\begin{array}{c}-0,2 \\
+\quad 1,1 \\
++\end{array}$ & $\begin{array}{c}+0,4 \\
-0,5 \\
\text { NS }\end{array}$ & $\begin{array}{r}2,2 \\
-1,2 \\
N S\end{array}$ \\
\hline
\end{tabular}

\section{II. - RÉSUitats ét DISCUSSION}

Les tableaux des annexes I et 2 (mesures sur carcasses entières des agneaux Berrichon et Southdown) rapportent les moyennes, les écarts types et les différences des estimées $\mathrm{R}, \mathrm{M}_{j}, \mathrm{~S}_{k}$ et $\mathrm{A}_{l}$ pour chacune des mesures. Nous y avons joint les résultats des tests $\mathrm{F}$ de signification des effets contrôlés. Préalablement à l'étude de l'effet de la race du père sur les critères de qualité des carcasses, nous analyserons l'intervention du poids à l'abattage, de l'année, du sexe et du mode d'élevage des agneaux. 


\section{Effet du poids à l'abattage sur les caractéristiques des carcasses}

Bien que la variation du poids à l'abattage ne dépasse pas $2 \mathrm{~kg}$ autour de la moyenne (abattage à poids constant) il a été possible de déceler des variations dues à ce facteur. Le poids à l'abattage semble intervenir pour une grande part dans les variations du poids de la carcasse, du coffre et du baron. Par ailleurs la hauteur de poitrine (Th), la largeur de poitrine (Wr) et la largeur aux gigots (G) sont presque toujours en liaison significative avec le poids à l'abattage. BocCARD, IJUMONT et

ANNE

Mensurations (moyenne, écart-type, test $\mathrm{F}$ de signifi

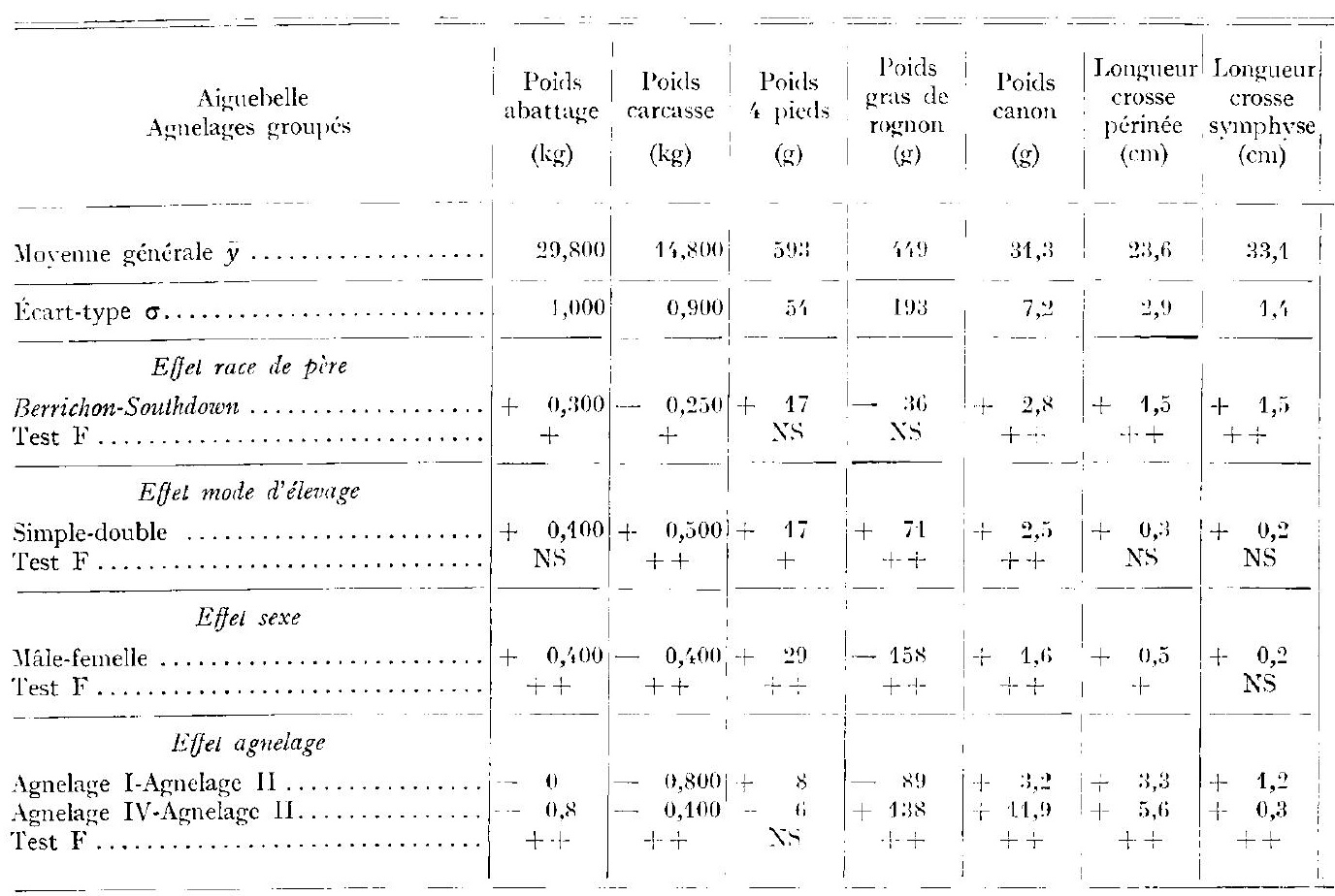

NS : Effet non significatif $(\mathrm{P}>0,05)+$ Effet significatif $(0,05>\mathrm{P}>0,01)$.

++ : Effet hautement significatif $(\mathrm{P}<0,01)$. Table de Snellecor.

PEYron (I964) montrent à la suite d'autres auteurs que ces mesures sont en liaison avec le poids de la carcasse de façon moins étroite cependant que le tour de poitrine (U).

Les animaux les plus lourds tendent à avoir un gras de rognon plus développé. Mais aucune liaison n'est discernable pour le gras de couverture dont la mesure a certainement été déterminée avec une moindre précision. Les mesures d'importance de 1 'os sont rarement en liaison significative avec le poids à l'abattage. I1 semble donc que même dans l'intervalle étroit considéré, la part due au développement du tissu 
adipeux dans un accroissement de poids est plus grande que celle revenant au tissu osseux dont le développement a commencé plus précocement.

La longueur du gigot $(\mathbf{F})$ n'est pas en liaison avec le poids à l'abattage, même dans le cas des agnelages de Marmilhat pour lesquels l'éventail des poids est plus ouvert. Boccard, Dumont et PEYRON (I964) n'ont trouvé eux aussi qu'une faible liaison entre cette mesure de conformation et le poids des carcasses sur une étendue de poids beaucoup plus grande.

ation des effets principaux, valeur de $b$ )

\begin{tabular}{|c|c|c|c|c|c|c|c|c|c|c|}
\hline $\begin{array}{l}\text { ongueur } \\
\text { queue } \\
\text { con } \\
\text { (cm) }\end{array}$ & $\begin{array}{l}\text { Largeur } \\
\text { gigot } \\
\text { (cm) }\end{array}$ & $\begin{array}{l}\text { Epaisseur } \\
\text { gigot } \\
(\mathrm{cm})\end{array}$ & $\begin{array}{l}\text { Ianteur } \\
\text { poit rine } \\
(\mathrm{cm})\end{array}$ & $\begin{array}{c}\text { Largeur } \\
\text { poitrine } \\
(\mathrm{cm})\end{array}$ & $\begin{array}{l}\text { Largenur } \\
\text { aux } \\
\text { çpatules } \\
\text { (cm) }\end{array}$ & $\begin{array}{l}\text { Largeur en } \\
\text { arricre des } \\
\text { épaules } \\
\text { (cm) }\end{array}$ & $\begin{array}{c}\mathrm{O}_{S_{1}} \\
\text { épaisseur } \\
\text { crosse } \\
(\mathrm{mm})\end{array}$ & $\begin{array}{c}O_{s_{2}} \\
\text { epaisseur } \\
\text { condyle } \\
\text { (min) }\end{array}$ & $\begin{array}{l}\text { Indice } \\
\text { "Os" } \\
(\mathrm{mmm})\end{array}$ & $\begin{array}{c}\text { II } \\
\text { Longueur } \\
\text { canon } \\
\text { (mm) }\end{array}$ \\
\hline $6^{\prime}+$ & 21,0 & 11,8 & 23,4 & 20,9 & 18,9 & 15,8 & 25,9 & 36,6 & 61,8 & 61,9 \\
\hline 2,7 & 1,0 & 0,9 & $0, !$ & 1,3 & 1,6 & 0,9 & $\because, 0$ & $t_{1}, 2$ & 4,7 & 11,9 \\
\hline $\begin{array}{r}2,0 \\
++\end{array}$ & & $+\underset{N S}{0,2}$ & $\begin{array}{r}+0,5 \\
++\end{array}$ & $\begin{array}{r}-0,2 \\
\text { NS }\end{array}$ & $\begin{array}{r}-0,5 \\
++\end{array}$ & $\begin{array}{r}-0,1 \\
N S\end{array}$ & $\begin{array}{r}+1,0 \\
++\end{array}$ & $\begin{array}{c}+1,2 \\
++\end{array}$ & $\begin{array}{r}+2,2 \\
++4\end{array}$ & $\begin{array}{c}+\begin{array}{c}1,3 \\
+\end{array} \\
++\end{array}$ \\
\hline $\begin{array}{l}0,7 \\
+\end{array}$ & $\begin{array}{r}-0,1 \\
\text { NS }\end{array}$ & $\begin{array}{r}+0,3 \\
++\end{array}$ & $\begin{array}{r}0,1 \\
\times S\end{array}$ & $\begin{array}{c}-0,5 \\
+\end{array}$ & $\begin{array}{r}-0,1 \\
x-5\end{array}$ & $\begin{array}{r}0,3 \\
\times S\end{array}$ & $\begin{array}{l}+\quad 0,2 \\
1\end{array}$ & $\begin{array}{r}0,1 \\
x\end{array}$ & $\begin{array}{c}0,3 \\
\times\end{array}$ & $\div \underset{x}{2}$ \\
\hline $\begin{array}{l}0,3 \\
\text { NS }\end{array}$ & $\begin{array}{c}0,1 \\
\mathrm{NS}\end{array}$ & $\begin{array}{c}-0,3 \\
+\end{array}$ & $\begin{array}{r}0,2 \\
\mathrm{NS}\end{array}$ & $\begin{array}{c}0,2 \\
N S\end{array}$ & 0 & $\begin{array}{r}0,4 \\
++\end{array}$ & $\mid \begin{array}{r}+1,0 \\
-+\end{array}$ & $\begin{array}{r}+1,3 \\
++\end{array}$ & $\begin{array}{r}+2,3 \\
\mathrm{NS}\end{array}$ & $\underset{\mathrm{NS}}{0,4}$ \\
\hline $\begin{array}{r}1,0 \\
2,6 \\
++\end{array}$ & $\begin{array}{c}+0,2 \\
-0,3 \\
+\end{array}$ & $\begin{array}{r}-0,1 \\
+\quad 0,7 \\
++\end{array}$ & $\begin{array}{r}-0,5 \\
+\quad 0,3 \\
++\end{array}$ & $\begin{array}{r}-0,8 \\
+\begin{array}{r}+3 \\
++\end{array}\end{array}$ & $\begin{array}{r}-3,5 \\
-0,2 \\
++\end{array}$ & $\begin{array}{r}-0,2 \\
+\quad 0,3 \\
++\end{array}$ & $\begin{array}{r}-2,1 \\
-\quad:, 0 \\
++\end{array}$ & $\begin{array}{r}-8,8 \\
-\quad 6,9 \\
++\end{array}$ & $\begin{array}{c}10,9 \\
-\quad 9,9 \\
++\end{array}$ & $\begin{array}{c}+\quad 1,7 \\
+\quad 16,5 \\
++\end{array}$ \\
\hline
\end{tabular}

Il semble donc que les répercussions du poids à l'abattage sur la qualité des carcasses concernent surtout l'état d'engraissement des carcasses. Toutefois notre préoccupation n'est pas ici d'étudier l'effet du poids à l'abattage sur les caractéristiques des carcasses mais seulement d'éliminer cette source de variation : cette méthode de calcul a pour conséquence d'attribuer au poids de carcasse, la valeur d'un rendement $\left(=\frac{\text { poids de carcasse }}{\text { poids à l'abattage }}\right)$

Annales de Zootechnie. - I967. 


\section{Effet du sexe}

Le poids des carcasses femelles est supérieur en moyenne de 200 à $600 \mathrm{~g}$ à celui des carcasses mâles, bien que les mâles aient été abattus à un poids supérieur aux femelles de o à $750 \mathrm{~g}$ selon les agnelages. Malgré cette supériorité en poids des carcasses femelles, les mesures des tissus osseux tant en épaisseur que pondérales sont plus élevées chez les mâles (différences hautement significatives) : poids de 1'os canon ( $\mathrm{I}, 2$ à $3,5 \mathrm{~g})$, poids des 4 pieds $(28,5$ à $57,4 \mathrm{~g})$, épaisseur de la crosse $(0,9$ à $\mathrm{I}, 8 \mathrm{~mm})$, épaisseur du condyle ( $\mathrm{I}, 3$ à $2,2 \mathrm{~mm}$ ).

Le surcroît de poids des carcasses femelles se manifeste au contraire par un tissu adipeux plus important : les écarts avec les carcasses mâles variant de 47,5 à $202 \mathrm{~g}$ pour le poids de gras de rognon, et de $\mathrm{I}, 0 \mathrm{~mm}$ à $\mathrm{I}, 4 \mathrm{~mm}$ pour l'épaisseur du gras de couverture.

Les carcasses mâles ont en général des rayons osseux plus longs que les femelles mais les écarts ne sont pas toujours significatifs. Les résultats des analyses sur les autres mesures permettent aussi de dire que les carcasses femelles sont en moyenne plus larges que les carcasses mâles (largeur de poitrine et largeur aux épaules) avec des gigots plus épais (différences non significatives).

Beaucoup de travaux réalisés sur les ovins ont montré que le développement des agneaux femelles se fait plus précocement que celui des agneaux mâles. Tandis que les mâles ont en général une vitesse de croissance supérieure à celle des femelles (Palsson et Verges, I952 ; Desvignes, CatTin-Vidal et Poly, ig65), la conformation recherchée sur le marché et l'état d'engraissement souhaité, sont atteints pour les agneaux femelles à un poids de carcasse inférieur.

Palsson et Verges (I950), Clarke et McMeEkAN (I952) montrent qu'à poids constant les carcasses femelles ont des os plus légers avec un poids d'os canon plus faible. La plus grosse différence se manifeste pour le tissu adipeux plus important en pourcentage et en épaisseur dans la région dorsale Il en résulte que ces carcasses obtiennent de meilleures notes d'appréciation de développement du gras dans les travaux de HAZEL et 'TERRIIL (I946) et de WALKER (I952) concernant les marchés anglo-saxons. HINER et 'THORNTON (I 662) trouvent aussi un effet sexe hautement significatif sur la composition tissulaire du train de 9 côtes représentatives de la composition globale de la carcasse.

En ce qui concerne la conformation, McLEAN (I948), LARGE et TAYLER (I954), KING, Wa'son et Young (I959) montrent qu'au même âge les agneaux femelles ont un os canon plus court. LARGE et TAYLER (I955) calctlent un rapport largeur aux ilions/longueur du métacarpe plus élevé pour les femelles. WALKER (I95I) constate aussi que les agneaux néo-zélandais femelles ont une meilleure conformation que les agneaux mâles au même âge.

Les résultats obtenus dans cette expérience sont du même ordre puisque à poids d'abattage constant, les carcasses d'agneaux femelles sont plus lourdes (meilleur rendement), plus légères en os et plus chargées en gras, et tendent à avoir une meilleure conformation. Ces résultats sont aussi à rapprocher de ceux de Boy LAN et SEALE (I965) obtenus sur des carcasses d'agneaux abattus à poids constant (meilleur rendement et développement plus important du gras dorsal des carcasses femelles). Notons que comme ces auteurs, nous ne mettons en évidence aucun écart mâlefemelle pour les dimensions de la section du muscle longissimus dorsi. 


\section{Effet du mode d'élevage ${ }^{(1)}$}

L'effet dı mode d'élevage est essentiellement un effet " maternel » se traduisant par une vitesse de croissance moyenne inférieure des agneaux doubles par rapport aux agneaux simples (1)Esvignes, CatTin-Vidal, Poly, Ig65). Cet effet, important aux alentours de la naissance et durant le premier mois, s'estompe avec l'âge (MAson et Dassat, I954; Huxter. I956; Harringtox, Whitemax et Morrison, I958). Il est encore une source importante de variation pour le poids au sevrage, pour la conformation et l'état d'engraissement des carcasses (HAZEI. et TERRILI, I946; Sidwell, et al., I95I ; DE BACA et BogarT, I959). Paissox et Verges (I952) soulignent que les écarts de poids sont moins prononcés lorsque le niveau énergétique alimentaire est maintenu élevé durant la gestation et la période d'allaitement.

L'effet du mode d'élevage se manifeste à Aiguebelle et à Marmilhat sur le poids des carcasses, sur le poids de l'os canon et dans une moindre mesure sur le poids du gras de rognon.

Le poids des carcasses des agneaux élevés simples est toujours plus élevé que celui des agneaux élevés doubles (de I 30 à 7 Io g), les écarts étant presqute toujours significatifs. Le rendement des agneaux simples est donc plus élevé que celui des agneaux doubles (carcasses plus lourdes à poids d'abattage égal). L'écart entre les poids moyens des carcasses "simples" et "doubles " peut être chì à un tissu gras plus important chez les agneaux simples bien que la différence ne soit pas significative (50 à $90 \mathrm{~g}$ pour le gras de rognon à Aiguebelle). Ce meilleur rendement des carcasses d'agneaux simples intervient malgré un poids de l'os canon et les 4 pieds supérieur. BocCARD et IJUPIAN (I96I) ont déterminé que les carcasses des agneaux à croissance rapide ( $35^{\circ} \mathrm{g}$ par jour) avaient un développement adipeux plus important que les carcasses d'agneaux à croissance lente (généralement bessons).

\section{Effet de l'année et de l'élerage}

L'effet de l'année a été contrôlé lors de la comparaison des croisés Southdoron aux croisés Berrichon, et ne porte donc que sur ces 2 groupes d'agneaux totalisant 328 carcasses. Il n'a pas été fait de comparaison statistique entre les deux élevages.

I)ans l'élevage d'Aiguebelle l'effet de l'année est hautement significatif pour l'ensemble des variables mesurées, ce qui traduit une forte influence des facteurs de milieu. Au contraire dans l'élevage de Marmilhat (dont les agnelages successifs ont été cependant très divers en poids moyens) l'effet de l'année se manifeste seulement sur les mesures d'importance de l'os (le poids du gras de rognon n'a pu faire l'objet d'une analyse à Marmilhat).

Paisson et Verges (I950), Krng, Watson et Young (I959), Boccard et DUPIAN (I96I) ont étudié la composition tissulaire de carcasses d'agneaux amenés au même poids avec des vitesses de croissance différentes. Ils ont montré que la composition tissulaire des carcasses et la répartition anatomique des tissus était affectée par le régime alimentaire. Le tissu adipeux paraît être le plus sensible aux conditions de milieu, son développement, particulièrement dans la région dorsale,

(1) Mode d'élevage a simple ": agnealux nés simples ou doubles élevés simples. Node d'élevage " double " : aqneaux nés doubles élevés doubles. 
dépendant du niveau énergétique de la ration au cours des dernières semaines avant l'abattage. Il est remarquable ainsi, dans notre expérience, que des carcasses de même type sont plus chargées en gras de rognon et en gras dorsal à Aiguebelle oì le poids à l'abattage a été atteint plus tôt qu'à Marmilhat (ces différences peuvent être dues également à la nature génétique des femelles utilisées dans les 2 élevages). Dans l'élevage même d'Aiguebelle, les agneaux du premier agnelage dont la croissance a été plus lente, ont une épaisseur de gras clorsal inférieure de I mm, et un gras de rognon plus léger de roo g en moyenne en comparaison avec les agnelages suivants.

TABLEAU 3

Interactions de premier ordre testies pour l'ensemble des agnelages (croisés Berrichon et Southdow'n)

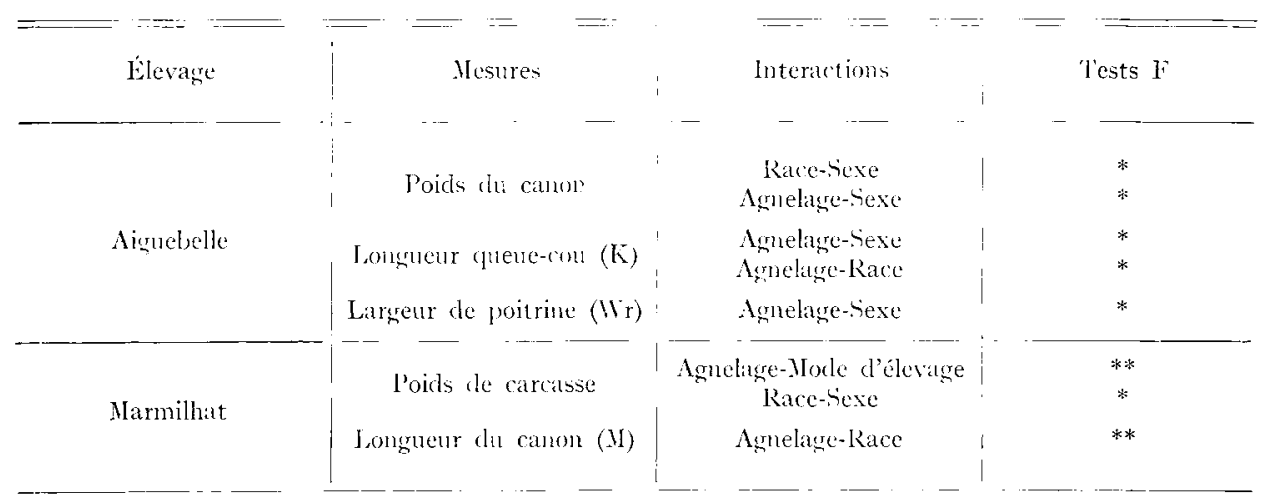

* Interaction simnificative $(0,05>\mathrm{P}>0,01)$.

** Interaction hatutement signilicative ( 1 < $<0,111)$.

Alors que les tests d'interaction ont permis cle vérifier l'hypothèse nulle exprimée dans le schéma mathématique d'analyse, et ceci pour tous les agnelages pris un par un, il n'en a pas été de même dans l'analyse des agnelages groupés. I a valeur du test $\mathrm{F}$ d'interaction a dépassé le senil de probabilité inférieure à 5 p. Ioo pour un certain nombre de variables (à Aiguebelle pour le poids clu canon, la longueur queuecou et la largeur de poitrine, et à Marmilhat pour le poids de carcasse et la longueur du canon). Le test d'ANDERSON et BANCROFT a fait apparaitte les interactions de premier ordre rapportées dans le tableau 3.

L'interaction agnelage-mode d'élevage pour le poids de carcasse à Marmilhat peut s'expliquer par les conditions particulièrement sévères d'alimentation pour le $3^{\mathrm{e}}$ agnelage qui ont entraîné une accentuation de l'effet du mode d'élevage sur la vitesse de croissance et le poids de la carcasse par rapport aux autres années.

Les autres interactions observées (essentiellement agnelage-sexe et agnelagerace de père) tendent à nous indiquer que d'une année à l'autre. des animaux de même sexe ou de même nature génétique ne réagissent pas de la même façon à des conditions de milieu différentes. Ces interactions n'interviennent cependant pas sur des mesures dont les répercussions sur la qualité des carcasses sont réelles. Elles n'ont 
donc pas d'importance économique. KIxG, WA'ssox et Young (I959) étudient plusieurs mensurations sur des carcasses d'agneaux croisés soumis à des régimes alimentaires différents. Ils ne déterminent une interaction génotype-milieu que sur le poids de l'os canon. Les auteurs concluent qu'il n'est pas nécessaire de placer dans un milieu déterminé les races et croisements que l'on désire comparer sur leur aptitude à produire un certain type de carcasse.

\section{Effet de la race du pire}

Ayant fait la part des différents facteurs (poids à l'abattage, sexe, mode d'élevage et année). nous pouvons isoler plus facilement l'effet des races de béliers utilisés sur les critères de qualité des carcasses.

La pratique du croisement industriel a en particulier pour objectif 1'amélioration de la qualité des carcasses produites. Cette amélioration peut concerner la conformation, mais peut intervenir aussi sur la composition tissulaire, ce dernier facteur, économiquement négligé actuellement par rapport à la coquetterie de la carcasse, n'étant pas le moins important pour le consommateur.

Une précédente revue bibliographique ( $\mathrm{F}_{\text {LAMANT }}$ et BoccARD, I966) a rassemblé les résultats d'un certain nombre cle travaux qui tendent à montrer l'indépendance de la composition des carcasses en morceaux par rapport à leur conformation. Quant à la composition tissulaire, elle est susceptible de variations qui déterminent la quantité de viande consommable à poids de carcasse constant. L'intérêt économique porté jusqu'à présent à la conformation explique qu'un grand nombre de travaux aient eu pour objet l'étude de ce caractère. De plus, les mesures représentatives telles que longueur du canon et longueur du gigot, ont manifesté des héritabilités relativement élevées (McLean, I948 ; RaE, I948; 'Taneja, I958; Hillman et al., ig62 ; GalaL, CARTWRIGHT et Sheitov, I965). Une sélection peut donc être facilement entreprise sur la longueur de gigot, par exemple. Les résultats de contrôle de descendance intraraces indiquent que les différences observées entre béliers se transmettent pour une large part attx descendants (Mcliax, I948; Barton, Phirims et Ciarke, ig49; CoI EMAN, I95I).

L'utilisation de béliers cle races améliorées pour la conformation, en croisement avec des brebis de races moins bien conformées mais rustiques et présentant de bonnes qualités maternelles, permet en outre d'obtenir en une génération des agneaux dont la conformation se rapproche de celle des pères (MILLER et DAMIEY, I95I; Hariag, Ieydol.ph et Schoize. I954 ; Suck, I957 ; SkJERvold et GJEdrem, I958 ; Schmidt, Ig60). Les carcasses de ces produits sont susceptibles d'être classées dans une très bonne qualité sur le marché si le père a été bien choisi. Parmi les races utililisées, les béliers Southdown se distinguent par une conformation de leur descendance supérieure à celle obtenue avec les béliers d'autres races (PAI,SSON, I940; CoOP et CLARKE, I957 ; DE BACA et BOGART, I959).

Du point de vue de la composition tissulaire on constate que, à poids constant, les carcasses plus " compactes" sont plus chargées en gras que les carcasses moins bien conformées. Corrélativement le poids des os est plus faible (Barton, Philitips et ClaRke, I949; SCHEELJE, I959). Le plus souvent les croisés Southdown ont des os plus légers et un gras plus développé (WAIKER, I949). 
Les expériences d'Aiguebelle et de Marmilhat permettent de comparer les carcasses de race pure Préalpes et Bizet aux carcasses croisées Southdoren et Berrichonne du Cher plus particulièrement.

Elles conduisent aux remarques suivantes :

a) Poids de la carcasse.

Le poids moyen des carcasses n'est influencé que très peu par la race du père. Cependant les carcasses Préalpes pures sont souvent les plus légères dans l'élevage d'Aiguebelle, où les carcasses croisées Southdown ont un meilleur rendement que les carcasses croisées Berrichonne du Cher. Par ailleurs, aucune différence nette n'intervient en faveur de l'un des types de carcasses pour le poids du baron.

b) Développement du gras.

I) fait de grandes variations intra-groupes, le poids du gras de rognon et l'épaisseur du gras dorsal sont peu souvent déterminés par la race du père. Les carcasses croisées Berrichonne $d u$ Cher tendent à être moins grasses que les carcasses croisées Charmoise et Southdor'n: elles se classent donc pour ce critère en position intermédiaire par rapport aux carcasses de race pure Cette aptitude peut donc constituer un avantage pour les béliers Berrichon $d u$ Cher compte tenu des exigences du marché français.

c) Développement osseux.

La variabilité entre races de pères se manifeste beaucoup plus pour le poids de l'os canon, représentatif de l'importance du squelette dans la carcasse.

Corrélativement à leur plus faible état d'engraissement, les carcasses de race pure présentent un tissu osseux plus important que les carcasses croisées Southdou'n et Charmoise. Mais les os des croisés Berrichonne du Cher, sont plus lourds et plus épais (écarts hautement significatifs) que les os des croisés Southdou $n$, et très proches, quoique plus légers, des carcasses de races pures Préalpes ou Bizet.

\section{d) Longueur des rayons ossenx et conformation des carcasses.}

Les agneaux croisés ont toujours en moyenne des rayons osseux plus courts que les agneaux de race pure. Les croisés Southdoren et Charmoise obtiennent la meilleure conformation, alors que les gigots des croisés Berrichon du Cher ont une longueur intermédiaire entre celle des croisés. Southdown et celle des agnea11x de race pure.

e) Autres mesures.

I)ans la plupart des agnelages, les carcasses de race pure Préalpes et Bizet sont plus étroites que les carcasses croisées. Les autres mesures ne révèlent aucun effet de la race des béliers, en particulier le développement musculaire ne présente pas de différences importantes au niveau du muscle longissimus dorsi. Il est possible que les plus grandes dimensions des carcasses croisées Berrichonue du Cher d'Aiguebelle (longueur du corps, hauteur de poitrine, largeur de poitrine) s'expliquent par des différences entre les béliers utilisés à Aiguebelle et ceux utilisés à Marmilhat

La pratique du croisement industriel se manifeste donc sur la qualité cles carcasses essentiellement par une meilleure conformation des produits (gigots plus 
courts, et dans une moindre mesure, carcasse plus large). Ces résultats sont en accord avec les travaux rapportés plus haut. Chez les ovins les 2 principaux critères de qualité des carcasses (longueur du gigot et développement du tissu adipeux) apparaissent généralement en liaison négative. Cette corrélation décrite par HAMmoNi) (I932) peut être en fait la conséquence d'une sélection menée parallèlement sur ces 2 caractères, tout particulièrement chez les races 1 owon anglaises qui orientent depuis déjà plus d'un siècle la production mondiale d'agneaux de boucherie. Il n'est pas interdit de penser qu'un matériel animal différent puisse conduire à un résultat différent. Nous avons ainsi l'exemple du phénomène "culard " dans l'espèce porcine (race de Piétrain: KNOER'tzer, I96r ; Oldivier, rg66) et dans l'espèce bovine (1,AUVergne, Vissac, et Perramon Ig63) oì la compacité de l'animal ne s'accompagne pas d'un tissu adipeux important.
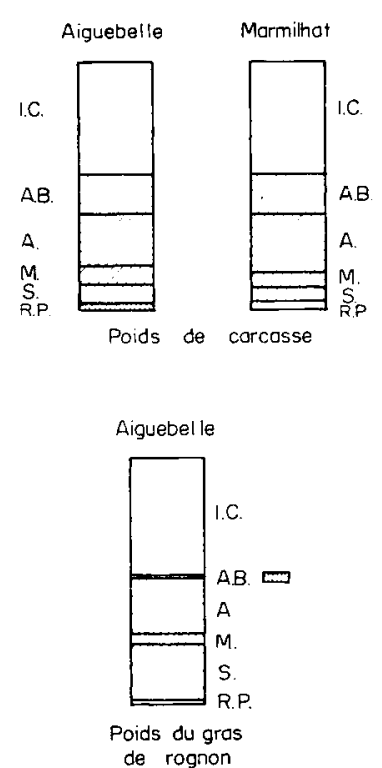
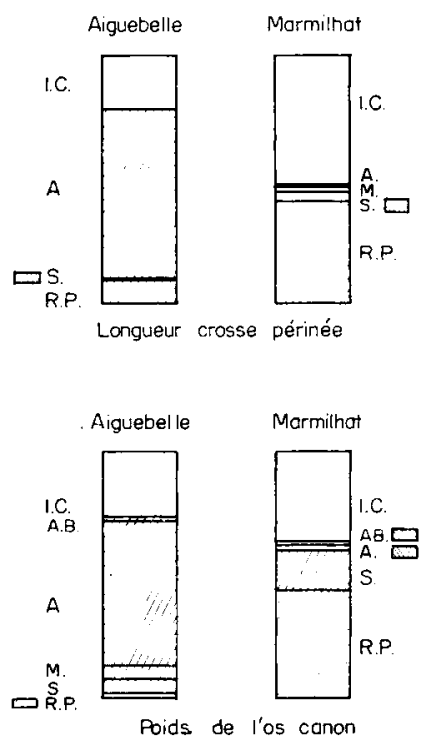

FIG. 2. - Part relative des principan facturs su la variation du poils de carcasse, de la longupur crosse-périnée, du poids de gras de rognon et du poids du canon.

I. $C$ : Variation intra-cellules; $A B$ : Effet du poids a l'abattage ; $A$ : Effet de l'anné ; II : liffet du mode d'élevage; $\mathrm{S}$ : Effet clu sext': R. I'. Effet de la race du pére.

Divili liffet hautement significatif ( $\left.\mathrm{I}^{\prime}<0,01\right)$.

WRT liffet significatif $(0,05>\mathrm{P}>0.01)$.

Les résultats obtenus dans notre expérience de croisements à Aiguebelle et Marmilhat vérifient la liaison biologique traditionnellement décrite puisque les agneaux croisés Berrichon, intermédiaires pour la longueur des gigots entre les agneaux de race pure et les agneaux croisés Southdown, le sont aussi pour le développement du tissu gras.

\section{Importance relative des différents facteurs}

Le sexe des agneaux, leur mode d'élevage et la race de leur père interviennent de façon différente sur les critères de qualités des carcasses. Aussi avons-nous déterminé la part de variation due à chacun de ces effets en pourcentage de la variance 


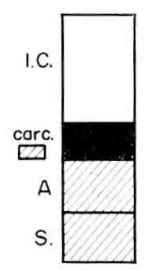

Poids du gras de rognon

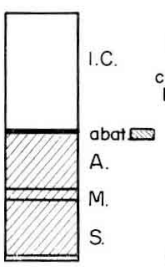

R.P.

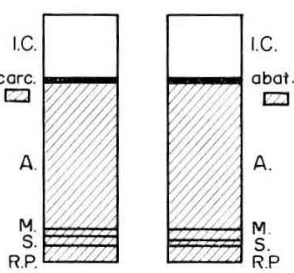

Poids du canon

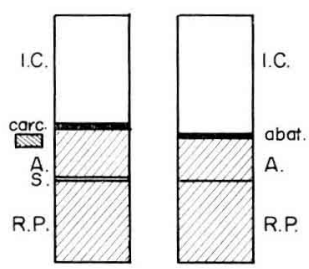

Longueur crosse symphyse

Fig. 3. - Comparaison des effets du poids à l'abatlage et du poids de carcasse sur le poids lu gras de rognon,

le poids de l'os canon el la longueur crosse-symphyse de's carcasses des agnelages de l'élevage d' tiguebelle.

I. C. : Variation intra-cellules; CARC : Effet du poids de carcasse; ABAT : Effet du poids a l'abattage ; A : Effet de l'année; II : liffet du mode d'élevage ; 5 : Lffet du sexe; R. l'. : Liffet de la race du père.

$$
\text { Mitil Effet hautement significatif }(P<0,01) \text {. }
$$

WDD Lffet significatif $\left(0,05>\mathrm{P}^{3}>0,01\right)$.
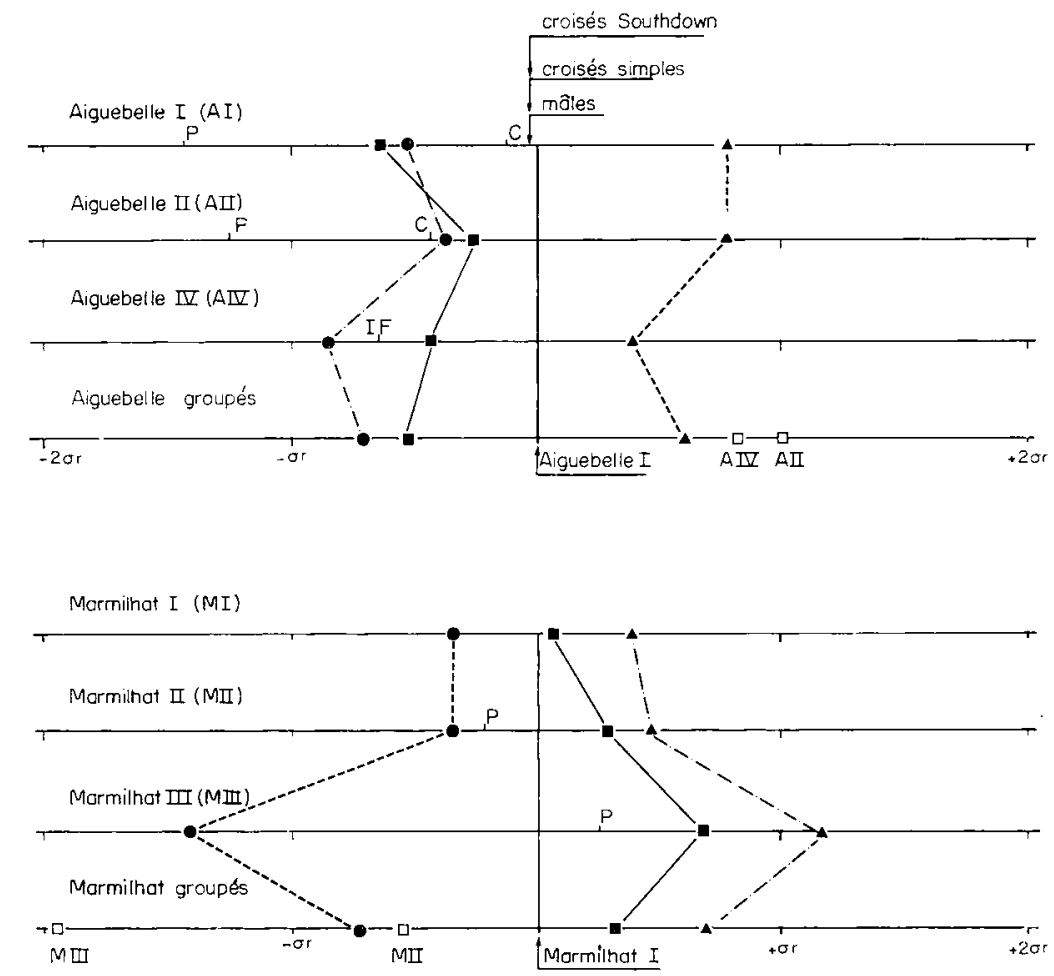

FIG. 4. - Estimécs des effets race du père, mode l'élerage, sexe el agnelage exprimées en unités d'écart-lype résiduel $\left(\sigma_{r}\right)$ pour le poids de la carcasse.

- Élevés doubles.

- Femelles.

- Croisés Berrichon.

C. Croisés Charmoise.

I. F. Croisés Ile-de-France.

P. Race pure. 
totale sur le poids de carcasse et sur 3 mesures particulièrement représentatives : la longueur crosse-périnée (indice de conformation), le poids de 1'os canon (indice d'importance du squelette) et le poids du gras de rognon (indice de développement du tissu adipeux). (fig. 2).

\section{a) Poids de carcasse.}

L'année constitue le facteur le plus important de variation du poids de carcasse. Par contre, l'influence de la race du père est très négligeable. Ainsi, les variations de rendement des carcasses sont beaucoup plus grandes entre sexe, mode d'élevage et année qu'entre races de père.
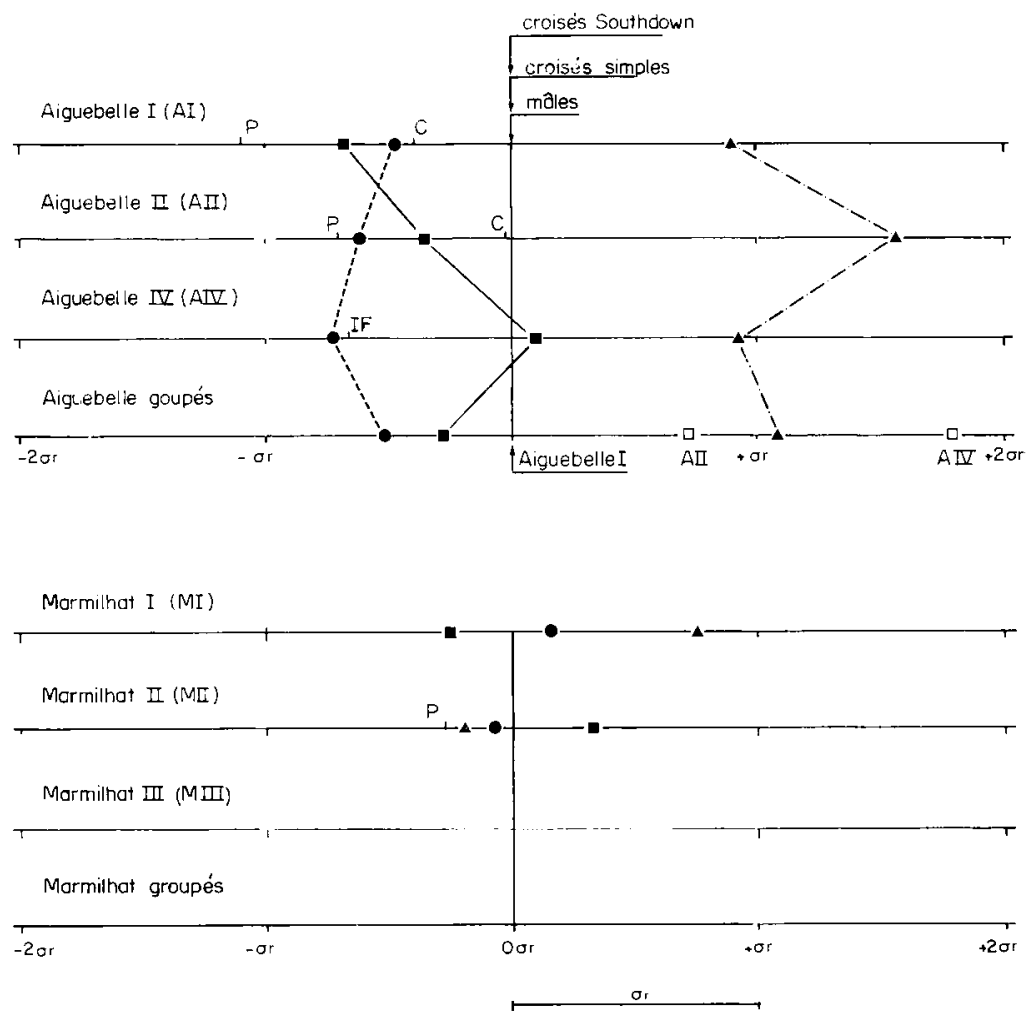

FIG. 5. - Estimées des effets race du père, mode d'élevage, sexe el agnelage exprimées en unités d'écart-lype résiduel $\left(\sigma_{r}\right)$ pour le poids du gras de rognon.

- Élevés doubles.

- Femelles.

- Croisés Berrichon.

C. Croisés Charmoise.

I. F. Croisés Ile-de-France.

P. Race pure.

b) Longueur du gigot.

Au contraire, pour l'élevage de Marmilhat les facteurs d'ordre génétique (race du père principalement, sexe accessoirement) déterminent la plus grande part (45 p. roo) des variations de conformation des carcasses (longueur crosse-périnée). Les autres facteurs n'ont qu'une faible influence. Ces résultats confirment les héri- 
tabilités généralement élevées calculées pour la mesure F. Pour l'élevage d'Aiguebelle l'influence de l'année est nettement prépondérante. Nous discuterons plus loin cette part importante prise par les facteurs de milieu dans cet élevage.
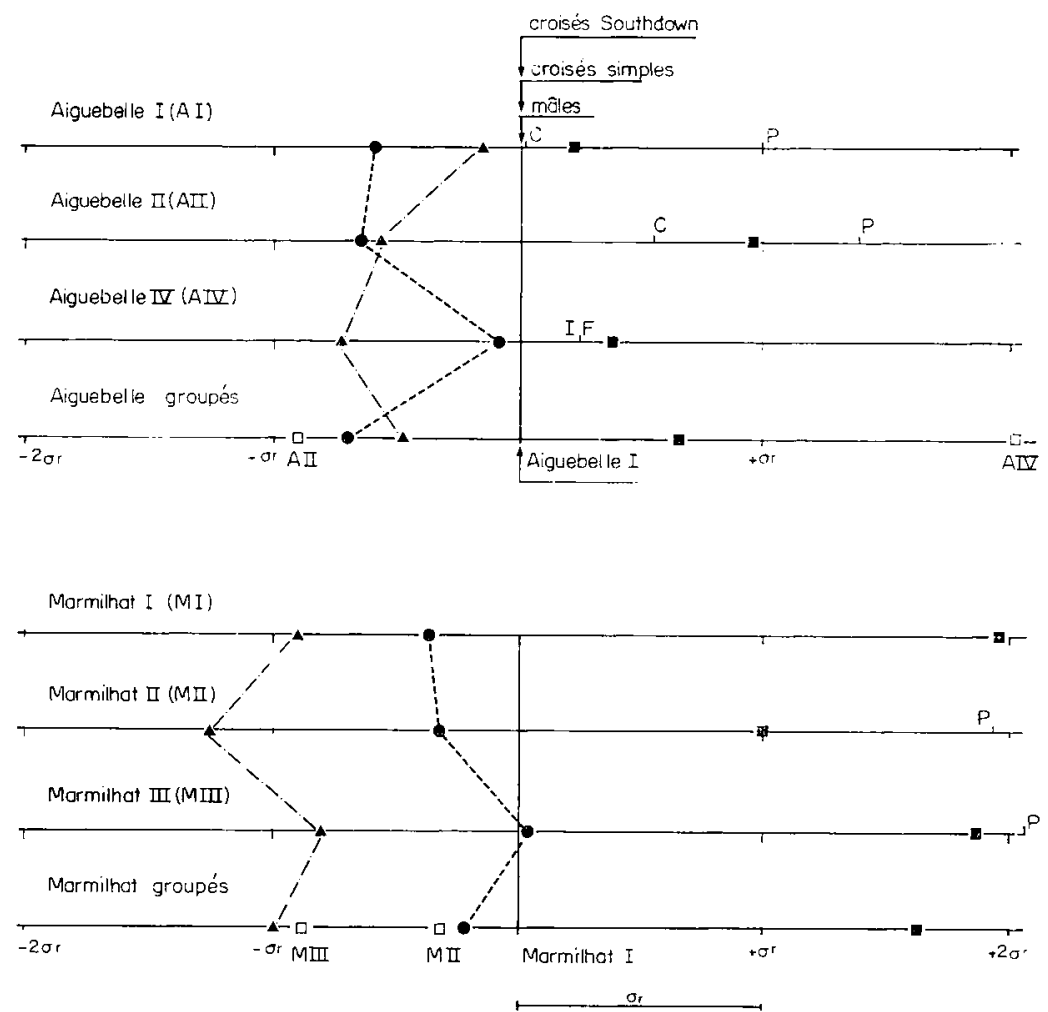

FIG. 6. - Estimées des effets race du père, mole d'élerage, sexe et agnelage exprimées en unités d'cart-lype résiduel $\left(\sigma_{r}\right)$ pour le poids de l'os cannt.

- Élevís cloubles.

- Femelles.

- Croisés Berrichon.

C. Croisés Charmoise.

I. F. Croisés lle-dte-linate.

P. Race jure.

c) Poids du gras de rognon.

Le facteur sexe joue avec le facteur année, le rôle le plus important dans les variations du poids du gras de rognon. Cependant la variance résiduelle estimée est encore de 1'ordre de 50 p. roo. Aussi dans le but d'éliminer des sources individuelles de variation avons-nous repris l'analyse de ce caractère en utilisant le poids de carcasse comme covariable à la place du poids à l'abattage dans le schéma mathématique. Ce notveau calcul concerne d'ailleurs les 3 mensurations retenues et a été effectué sur les données regroupées des 3 agnelages d'Aiguebelle (agneaux croisés Southdoan et Berrichon du Cher).

Le fait de tenir compte du poids de carcasse ne modifie pas les résultats des tests de signification des effets. Des différences se manifestent, par contre, dans la 
part relative des facteurs contrôlés dans la variation totale, et ceci essentiellement pour le gras de rognon : le poids à l'abattage rend compte de I p. Ioo des variations, alors que la part revenant au poids de carcasse atteint I5 p. Ioo (fig. 3).

Cette liaison plus importante obtenue pour le gras de rognon avec le poids de la carcasse, peut être rapprochée du rapport existant entre le rendement d'une carcasse et l'importance du tissu gras.
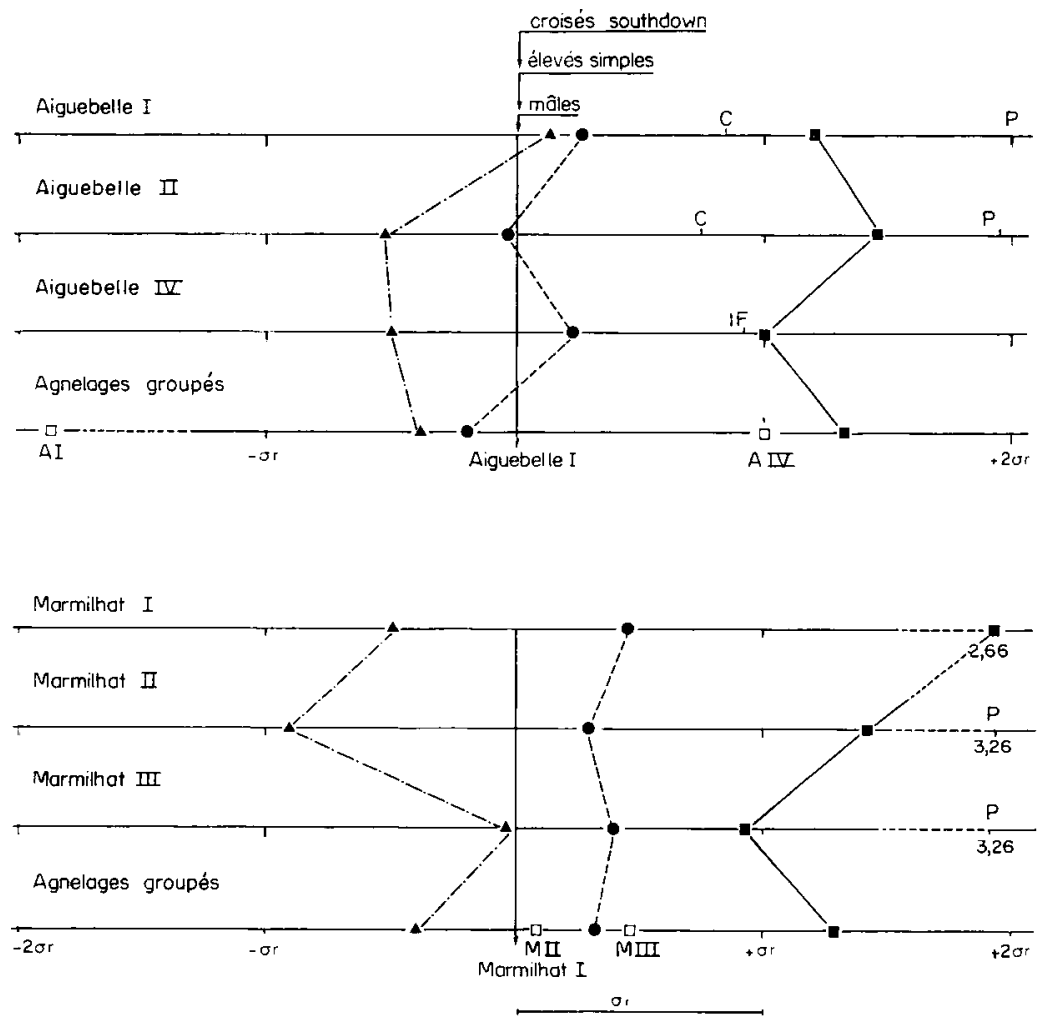

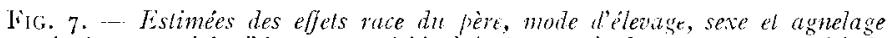
cxprimces en unilés d'écart-lype résiduel $\left(\sigma_{r}\right)$ pour la longucur crosse-périnée.

- Iiluvés doubles.

- Fumelles.

- Croisés bemichun.

C. Croisés Chammoise.

I. l. Croisés /le-de-Iranate.

I'. Rave pure.

d) Poids de l'os canon.

L'étude du poids de l'os canon fait apparaitre, conme pour la longueur du gigot, un effet différentiel des divers facteurs entre les élevages d'Aiguebelle et de Marmilhat. Ires facteurs génétiques (race du père et sexe) sont nettement plus déterminants à Narmilhat qu'à Aiguebelle. Cette différenciation se manifeste aussi pour d'autres mesures d'os quoique de façon moins nette. Les résultats obtenus en grou- 
pant les 3 agnelages d'Aiguebelle d'une part, et les 3 agnelages cle Marmilhat d'autre part, montrent une prépondérance de l'effet année à Aiguebelle et une prépondérance de 1'effet race du père à Marmilhat (fig. 2).

L'effet année important trouvé à Aiguebelle pourrait s'expliquer par les conditions de milieu différentes entre différents agnelages (I agnelage d'été, I agnelage de printemps, et I agnelage d'automne). Cependant à Marmilhat les résultats des agnelages furent également très différents les uns des autres bien que ce soient tous des agnelages d'automne.

Une autre explication est d'ordre génétique, la différenciation entre Aiguebelle et Marmilhat pouvant avoir pour origine la nature différente des races de brebis utilisées dans chacun des 2 élevages. Il faudrait alors admettre que les brebis Préalpes atténuent chez leurs descendants les différences d'ordre génétique présentes chez les béliers en ce qui concerne la conformation de la carcasse et le poids du squelette alors que les brebis Bizet permettant une plus grande expression des caractères des races des pères.

Nous avons exprimé sur les figures $4,5,6,7$, les écarts dus à chacun des facteurs de classement sur le poids de carcasse, la longueur crosse-périnée, le poids de l'os canon et le poids du gras de rognon, respectivement. L,es axes sont gradués en unité d'écart-type résiduel (intra-agnelage pour chacune des variables). On a porté sur ces axes, à partir d'une même origine, les estimées des effets de chacun des facteurs dont les valeurs sont données par la résolution des systèmes linéaires.

\section{C. - - CONCL,USIONS GÉNÉRALES}

L'amélioration de la vitesse de croissance des agneaux et de la qualité bouchère de leurs carcasses pose le problème de la relation entre croissance et précocité. Si nous comparons des groupes d'animaux différant entre eux par des vitesses moyennes de croissance ayant pour origine des facteurs alimentaires (mode d'élevage) ou plus généralement des facteurs de milieu (année par exemple), nous constatons alors, comme dans notre expérience, qu'un taux de croissance plus élevé correspond, à poids égal, à des carcasses dont le pourcentage de tissus adipeux est supérieur.

Si par contre, nous considérons des différences de vitesses de croissance déterminées par des variations d'ordre génétique nous observons le phénomène contraire. Ainsi les agneaux mâles ont la meilleure vitesse de croissance et en moyenne leurs carcasses sont les plus maigres. FiEI,1) et al. ( I963) déterminent que les lots d'agneaux descendants de béliers Southdown à croissance rapide fournissent les carcasses les plus maigres à poids constant. Mais on peut se poser la question de la nature de cette meilleure vitesse de croissance : s'agit-il d'une aptitude propre ou d'une conséquence d'un plus grand format adulte?

A l'échelon d'une population, d'une race ou même d'un élevage, facteurs de milieu et facteurs génétiques interfèrent de telle façon que l'observation du résultat global ne permet pas d'aboutir à des conclusions valables et utiles pour la sélection quant à la nature de la liaison vitesse de croissance-qualité des carcasses. Ainsi SCHEELJE (I959) estime que les groupes d'agneaux d'une même race présentant les 
meilleures carcasses ont eu aussi le meilleur engraissement, et SHEITON et al. (I954) déterminent sur les agneaux descendants de brebis Rambouillet, une relation positive entre la vitesse de croissance et le classement des carcasses (les carcasses les meilleures ayant les notes les plus élevées). On peut interpréter ces derniers résultats par une extériorisation maximum des potentialités bouchères dans les meilleures conditions de milieu. Les facteurs d'environnement ayant une influence généralement prépondérante, il importe d'éliminer leurs effets si l'on désire obtenir une représentation génétique de la liaison vitesse de croissance-qualité des carcasses. A ce propos, RAE (I956) signale que la sélection des béliers sur le poids à la naissance ou à un âge déterminé ne permet pas de préciser sur quel caractère porte exactement la sélection: précocité plus grande permettant d'atteindre le stade adulte plus rapidement, ou format plus élevé permettant d'obtenir une vitesse de croissance moyenne plus importante durant une plus longue période? 'TAYIoR (I965) souligne qu'en fait la liaison génétique est négative entre croissance et maturité, et qu'une sélection pour un format plus élevé entraîne automatiquement une sélection pour une moindre précocité, et réciproquement.

La comparaison des résultats de l'utilisation des béliers Southdon: $n$ et Berrichon $d w$ Cher en croisement industriel a vérifié la liaison entre le format de la race paternelle et la vitesse de croissance des produits (I)isvignes, CatTin-Vidal et Pois, I 966), mais aussi avec la composition tissulaire des carcasses. 'Tout particulièrement les agneaux croisés Southdow'n, plus gras et moins osseux, sont beaucoup plus proches physiologiquement de leur état adulte que les croisés Berrichon dont la croissance a été plus rapide. Il est intéressant de noter que les agneaux de race pure Préalpes n'ont pas joint leur faible vitesse de croissance, égale à celle des croisés Southdown, à un développement important du tissu adipeux. La liaison entre vitesse de croissance des agneaux et maturité de développement des carcasses, observée intra-race en sélection, n'est donc pas étroite entre races puisque certaines d'entre elles peuvent posséder une aptitude particulière qui ne détermine pas obligatoirement leurs caractéristiques pour d'autres critères. L'utilisation de telles races présente alors un grand intérêt pour une amélioration génétique par croisement.

Les résultats de cette expérience conduisent à une conséquence pratique quant au choix de la race de béliers pour le croisement industriel. Le type de production recherché étant ici l'agneau lourd de $30 \mathrm{~kg}$ vif, doté d'une épaisseur de gras dorsal ne dépassant pas 3 millimètres. la plus grande vitesse de croissance des agneaux croisés Berrichon jointe à un développement adipeux satisfaisant (moins important en moyenne que celui des croisés Southdown), nous amène à retenir ce type de croisement pour nos races de femelles rustiques des zones montagneuses.

Reçu pour publication en janvier 1967 .

\section{REMERCIEMENTS}

Nous tenons à remercier les Pères de l'Abbaye d'Aiguebelle et le Directeur de l'École régionale d'Agriculture de Marmilhat qui ont mis leurs troupeaux ì notre disposition et nous ont aidés à réaliser cette étude; la Fédération nationale ovine pour son aide financière ; les techniciens et stagiaires qui ont participé à cette expérimentation et à l'interprétation des données. 


\title{
SUMMARY
}

\author{
COMPARISON OF TIE VALUE OF DIFEERENT TYPES OF INDUSTRIAL CROSSING \\ FOR TIIE PRODUCTION OF LAMIBS FOR MEAT. II. - CARCASS VALUE
}

Southdor'n and Berrichonne du Cher (occasionally lle-de-France and Charmoise) rams were mated with Prealpes and Bizet ewes (two range mountain breeds). These experiments carried on at Aigliebelle Abbey (I)ôme) and Marmilhat Agricultural school (Puy-de-I)ôme) enabled us to compare crossbred to pure bred I'realpes and Bizet lambs as for the production of $\mathrm{s} 5 \mathrm{~kg}$ carcasses.

Besides slaughter weight, carcass wieght and joints weight, ig distinctive measurements of conformation and tissue composition were nuade on 45 . lamb carcasses. The lambs'growth had been studied by Desvignes, ('ATTIN-VIDAl and POLY (Ig66).

The variation factors of these estimations (slaughter weight, sex type of birth, age, sire's breed) were stat istically handled by least squares analysis. Figure 2 shows the percent variance attributable to each factor for carcass weight, lenght of leg (F), kidney fat weight, and cannom boneweight ; figures 4, 5, 6, 7 show the values estimated by least squares analysis for the same criteria.

Fenale carcasses appear to have better output (carcass weight/slaughter weight), butter conformation, lighter skelet on and greater fat depth male carcasses. Though they have a heavier cannon bone, single-bred lambs have higher output because a greater fat depth.

Differences in the sire's breed mainly alters the offspring's conformation and bone development.

- Southdown (or Charmotse) rams give the best carcass conformations, lighter bones, but deep fatty tissues.

- Berrichonne du cher rams give carcass conformations and tissue composition scoring between pure-bred and Southdown sired lambs.

As the supply ought to be thin-fatted is $\mathrm{kg}$ carcasses, the use of Berrichonne du Cher or lle-leFrance rams is advisable when crossed with range mountain ewes.

\section{RÉFÉRENCES BIBL,IOGRAPHIQUES}

Axdersox R. L., Baxcrom T. A., 1052. Shatistinl them in research. Mac Grow Iill. New York.

Barton R. A., Phillies 'T. O., Clakke li. A., 1949. linfuence of sire on fat lamb quality. Proc. Vea. Zeal. Soc, anim. Proll, 9, 66-84.

Boccard R., Dumont B. L., Peyron C., ic) $5^{\circ}$. Valeur signilicative cle quelques mensurations pour apprécier la qualité des carcasses d'agneaux. 1 th . Meet. Europ. Weal Res. Workers., Cambridge., sept. 15. Igth.

Boccaro R., Duplas J. M., igor. Stude de la production de la viande chez les Ovins. III. Note sur l'inthence de la vitesse de croissance sur la composition corporelle des agneaux. Inn. Zoolech., 10, 31-38.

Boccari) R., Dumont B. L., ig6o. Note sur la mesure et la va riation de l'adiposité des carcasses d'agnealu. 6 Réunion des Instituts de Recherche sur les Viantes. I'trecht. 29 août-3 sept.

Boccarp) R., Duront B. L., Peyrox., ight. Ĺtude de la production de la viande chez les Ovins. VIII. Relations entre les dimensions te la carcasse d'agneau. Ann. Kootech., 13, 367-378.

Boylay W. J., SEALE M. F., ig65. Relationships and variation among certain lamb carcass traits. Canal. J. anim. Sci., 45, 37-43.

Clarke R., Mc Meekan (. P., 1952. New Zealand lamb and mutton. lart I. Anatomical characteristics of lamb and mutton carcasses. Yea Zeal. J. Sci. Tech. agric., 33, I-15, 2.4-28.

Coleman J. M., 195г. Fat lamb production : inheritance of fleshing qualities. Agric. Gas. M. S. W', 62, $58-60$.

CoOP I. E., Crakke R., I952. A comparion of breeds of ram for fat lamb production. New Zeal. J. Sci. Tech. agric., 34, $153^{-1} 7 \mathrm{I}$.

DE BACA R. C., BOGART R., 1959. Factors affecting condition and conformation of crossbred spring lambs. J. anim. Sci, 18, I094-1 Io2.

Desvignes A., Darpoux R., 1964. Valeur d'élevage des brebis utilisées pour le croisement industriel avec différentes races de béliers. Bull. tech. Ing. Serv. agric., 195, 3-11.

Desvignes A., Cattin-Vidal P., Poly J., ig66. Comparaison de la valeur de divers types de croisement industriel pour la production d'agneaux de boucherie. I. Croissance pondérale des agneaux. Ann. Zootech., 15, 47-66.

Field R. A., Kemp J. D., Varney W. Y., Woolfolk P. G., Derrickson C. M., ig63. Carcass evaluation of lambs from selected sires. J. anim. Sci., 22, 364-367. 
Fimamant J. C., Boccaris R., ig6r. Estimation de la qualité de la carcasse des agneaux de boucherie. Ann. Zoolech., 15, 89-113.

Galar. li. S. E., Cartwricht T'. C., Sherjon M., 1965. Relationships anong weights and linear measurements in sheep and heritability estimates of some of these measurements. J. anim. Sci., 24, 385-39I.

HARING F, LEYdolPH W., SciIOLZE F., I954. Lämmermastversuch mit verschiedenen Schafrassen und Kreuzungen, zugleich eine Frage zur Methodik der Schafmastleistungsprüfung. Züchtungskunde, 26, 25-33.

Harrington R. B., Wirtiman J. V., Horrison R. D., i958. Estimates of some sources of variation in the body weights of crossbred lambs at different ages. J. anin: Sci., 17, 743-75I.

Hazel L. N., Terribe C. E., 1946. Heritability of type and condition in range Rambouillet lambs as evaluated by scoring. J. anim. Sci., 5, 55-61.

IIllaman M., Wheat J. D., Menzies C., Mackintush I. L., Merkel R. A., igG2. Heritabilities of and correlations between carcass and live animal traits in sheep. (Abstr.). J. anim. Sci., 21, 973.

IIniver R. L., Thorcton J. W., ighz, Study of certain lamb and carcass quality factors. J. anim. Sci., 21, 5I I-5I 5 .

Hunter (i. L., 1956. The maternal influence on size in sheep. J. agric. 5ci., 48, 36-60.

Kempthorne I)., I952. The design and analysis of experimenls. John Wiley and sons. Tew-York.

Kin; J. W. B.. Watson J. H., Iorsg (;. 13., 1959. Genotype-environnent interactions in the wintering of lambs. J. agric. Sci., 53, i 56-17.

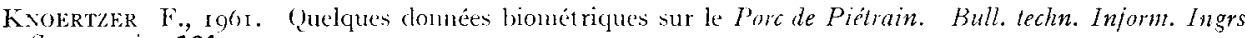
Serv. agric., 164, 3-37.

KRAMER C. Y., I950. Jxtension of multiple range tests to group neans with unequal numbers of replications. Biometrics, 12, 307-310.

LARGE R. V., 'lAyier J. C., 195t. Studies on the growth of Clun lambs. Limp. J. exper. Agric., 22, I 4 I-I 47 .

Lauvirgne J. J., Vissac B., P'rrranon A.. i963. Fitule du caratère cularl. 1. Mise au point bibliographique. Ann. Zootech., 12, I 3.3-I 56.

IicLeax J. W., I $9+8$. Progeny testing in sheep. The inheritance of birth weight, growth rate and camon bone length. Proc. New Zeal. Soc. anim. Prod., sth inn. Conf., s6-0 to.

Mason I. L., DAssat P., I95+. Milk, meat and wool production in the Langhe sheep of ltaly. Z. Tierz. Zcioht Biol., 62, 197-23+.

Miller K. P’.. DAltes D. 1.., I951. A study of crossbreeding sheep. I. anim. Sci., 10, 402-468.

Olivier L., ro66. Interactions entre génotype et milien d'engraissement chez les porcs de race $L_{\text {atrge- }}$ White, Vormande et de Piétrain. Ann. Zootech., 15, i7

Passiox. HI. 1939-1940. Meat qualities in the sheep, with sperial reference to Scottish breeds and crosses. 1. agric. Sci., 29, 560-30, I.

PALSSOX I1, VERGES J. B., I952. Liffects of the plane of nutrition on growth and developnent of carcass quality in lambs. I. 'The effects of high and low planes of nutrition at different agres. $J$. agric. Sci., 42, 92 .

PALSSON II., VERGES J. 13., I950. Liffects of the plane of nutrition on growth and development of earcass quality in lambs. II. Liffects on lambs of $30 \mathrm{lb}$ carcasse weight. I. agric. Sci., 42, 93-149.

RAF A. L., 1948. Some aspects of progeny testing in sheep. Proc. Verw Zeal. Soc. anim. Proil., 97-ro4.

SCHEelJE R., 1959. Auswertungen der Nachkommenschaftsprüfungen auf Mastleistung und Schlachtkörperqualität an Böcken des Merinosfleischafes in einer Testherde. 7iühuıngskunde, 31, 366-377.

Schump L., 1960. Kreuzungsversuche mit Merinolandschaten und Ile-de-linance. Bayer Landw. Jarhrb., 37,5 I $5-54$.

Sileiton M., Miller J. C., Magee W. 'T., HARDY W. T., I954. A summary of four years'work in ram progeny and performance testing. J. anim. Sci., 13, $215-228$.

Sibwell G. M., PRice D. A., GrandstafF J. O., 195 I. (ienetic and envirommental factors affecting type and condition in Vavajo and Vaqajo crossbred weanling lambs. J. aniw. Sci., 10, 45.-46r.

SKJERVOLD II., GJEDREM T., 1958. Kjottproduksjonsegenskapene hos tre saueraser of to krysningsgrupper. Meld. Norges Landb., 37, I-I8.

Starke J. S., JoukerT D. M., IgGi. A score card for lamb and mutton carcasses. J. agric. Sci., 57, 3I $9-323$.

Suck H. J., 1957. Untersuchungen an deutscheri schwareköpfigen Fleischschajen hinsichllich des Einflusses ron Original. Hampshire down - Böcken auf Wolleistung und Körperentwicklung in: zwei kurhessischen Stammherden. Institut für 'Tierzucht und Milchwirtschaft Justus Liebig Universität. Griessen. 59 1).

TANEJA G. C., I958. Heritabilities of body length, depth, width, fore camnon length, fold score, and mutton score in Australian Merno sheep. J. Genetics. 56, 103-109.

TAylor St. C. S., 1965. Pathways to maturity. Anim. Breed. Res. Org.. Report. January, 1965, $25-31$.

Walker D. E., I95I. The influence of sex upon carcass quality of New Zealand fat lamb. Nea Zeal. J. Sci. Tech. Agris., 32, 30-38. 